\title{
Male Prepuce
}

National Cancer Institute

\section{Source}

National Cancer Institute. Male Prepuce. NCI Thesaurus. Code C33049.

A fold of skin covering the tip of the penis. 